\title{
The arousal predisposition scale: Normative data
}

\author{
STANLEY COREN \\ University of British Columbia, Vancouver, Canada
}

\begin{abstract}
The arousal predisposition scale (APS) is a 12-item inventory that has been designed to measure individual differences in arousability. It has already been shown to predict patterns of sleep disruption, and task related arousal increases. Norms for the APS, based on data from 786 university students, are provided. Since the data indicate that there is a sex difference in arousability, with females showing higher APS scores, data are also tabulated separately by gender.
\end{abstract}

Arousal is an important component of theories of motivation, performance, attention, and personality, as well as an important concept in behavioral medicine and other branches of psychology. Much evidence, however, suggests that an individual manifests different degrees of arousal under varying conditions. This individual difference dimension of arousal may be viewed as a predisposition toward arousal, and we will call this trait arousability.

Research oriented toward the measurement of arousability has tended to take one of two main directions, emphasizing either physiology or self-report (see Kohn, Cowles, \& Lafreniere, 1987). The physiological approach involves the measurement of various physical indexes that may correlate with individual differences in arousability (see, e.g., Bodunov, 1985; Danilova, 1985; Uherik, 1985). The self-report approach has involved the attempt to assess subjective perceptions of arousal state, which seem to be highly correlated with the physiological measures (Thayer, 1967, 1970). Occasionally, both types of measurement have been incorporated as single dimensions in more complex assessment inventories, such as the neuroticism scale in the Eysenck personality inventory (Eysenck, 1970) or the strength of excitation scale in the Strelau temperament inventory (Strelau, 1983). Alternatively, individual differences in arousability may be scored as a composite of several scales, as in the EASI-III (Buss $\&$ Plomin, 1975). All of these self-report scales have been designed on the basis of theoretical constructs, personality typologies, or behavioral observations.

Rather than develop a scale based on theoretical constructs, Coren (1988) attempted to construct a measure

This research was supported in part by grants from the Natural Sciences and Engineering Research Council of Canada, the British Columbia Health Care Research Foundation, and the University of British Columbia. The author would like to acknowledge the assistance of Wayne Wong, Lynda Berger, Geof Donelly, and Dereck Atha, who assisted in the scoring of these data. Correspondence should be addressed to Stanley Coren, Department of Psychology, University of British Columbia, 2136 West Mall, Vancouver, BC V6T 1Y7, Canada. of arousability as a trait or a predisposition by using an empirical selection and validation procedure. Since current evidence suggests that several forms of insomnia may result from cognitive hyperarousal, he reasoned that the tendency toward disrupted sleep could be used as an index of arousability. Starting with an original pool of 314 items, he selected a 70-item preliminary inventory. Next, he tested a sample of 196 subjects in order to develop and validate a 12 -item self-report inventory that he called the arousal predisposition scale or APS. Item selection was based on the ability to predict a global measure of insomnia. The resultant scale was then cross-validated with a sample of 693 subjects, whereupon it was shown to be a valid and reliable predictor of six indexes of sleep disturbance. The resultant scale correlates 0.45 with a total sleep disruption score and has a split-half reliability of 0.83 .

Although the APS was developed as a measure of individual differences in arousability and validated against measures of insomnia, if the trait of arousability that it purportedly measures is generalizable, it may well be useful as a measure of individual differences in arousal that would be expected in various tasks and in the presence of environmental stressors. This has been confirmed in a recent study by Coren and Aks (1990), who were able to show that increases in arousal when an individual was asked to perform a task in the presence of distracting stimuli were correlated with APS scores.

Although the APS is an attractive instrument for the measure of arousability, involving only 12 items, and taking about 2-3 min to complete, its use has been limited somewhat, due to the absence of normative data against which an individual's score could be compared. To provide such norms, the following study was conducted.

\section{METHOD}

The arousal predisposition scale (Coren, 1988) was administered to 786 subjects (479 women and 307 men). The subjects were students enrolled at the University of British Columbia who participated on a volunteer basis. Mean age of the subjects was 20.1. 
Table 1

Norms for the Arousal Predisposition Scale

\begin{tabular}{|c|c|c|c|}
\hline APS Score & Total Sample & Women & Men \\
\hline $20^{*}$ & 0.6 & 0.0 & 1.6 \\
\hline 21 & 1.0 & 0.2 & 2.3 \\
\hline 22 & 1.7 & 0.2 & 3.9 \\
\hline 23 & 2.2 & 0.2 & 5.2 \\
\hline 24 & 3.6 & 1.3 & 7.2 \\
\hline 25 & 5.3 & 2.9 & 9.1 \\
\hline 26 & 7.6 & 5.4 & 11.1 \\
\hline 27 & 9.9 & 6.3 & 15.6 \\
\hline 28 & 12.1 & 7.3 & 19.5 \\
\hline 29 & 15.4 & 9.6 & 24.4 \\
\hline 30 & 19.6 & 12.9 & 30.0 \\
\hline 31 & 23.9 & 16.5 & 35.5 \\
\hline 32 & 29.1 & 21.5 & 41.0 \\
\hline 33 & 33.3 & 24.4 & 47.2 \\
\hline 34 & 39.8 & 31.3 & 53.1 \\
\hline 35 & 45.0 & 36.5 & 58.3 \\
\hline 36 & 50.4 & 42.0 & 63.5 \\
\hline 37 & 55.5 & 48.4 & 66.4 \\
\hline 38 & 61.7 & 55.5 & 71.3 \\
\hline 39 & 67.3 & 61.4 & 76.5 \\
\hline 40 & 72.1 & 66.8 & 80.5 \\
\hline 41 & 77.5 & 72.7 & 85.0 \\
\hline 42 & 81.7 & 78.3 & 87.0 \\
\hline 43 & 85.4 & 82.9 & 89.3 \\
\hline 44 & 88.8 & 87.1 & 91.5 \\
\hline 45 & 91.5 & 90.4 & 93.2 \\
\hline 46 & 93.6 & 93.3 & 94.1 \\
\hline 47 & 95.3 & 95.2 & 95.4 \\
\hline 48 & 96.9 & 96.7 & 97.4 \\
\hline 49 & 98.1 & 97.5 & 99.0 \\
\hline 50 & 99.1 & 99.2 & 99.0 \\
\hline $51 \dagger$ & 99.5 & 99.6 & 99.3 \\
\hline Total $N$ & 786 & 479 & 307 \\
\hline
\end{tabular}

Note-The APS scores are listed against the cumulative percentages for a total sample of 786 university students (mean age 20.1 years), with separate listings for men and women. *or lower. for higher.

\section{RESULTS AND DISCUSSION}

The distribution of APS scores is shown in the form of cumulative percentages in Table 1 . There was a difference in arousability between the men and the women. The women showed a mean arousability of 37.6 , which is 3.1 points higher than the men's average of 34.5. This difference is statistically significant $[t(784)=6.57, p<.001]$. Because of this sex difference in arousability, the men's and women's scores are also presented separately in Table 1 .

The normative data presented here should allow researchers who wish to use the APS in studies of arousability, insomnia, or personality, or in behavioral medicine or other areas, to interpret the scores of individual subjects more easily.

\section{REFERENCES}

Bodunov, M. V. (1985). Typology of mental activity as a temperamental trait and the level of activation of the nervous system. In J. Strelau, F.H. Farley, \& A. Gale (Eds.), The biological bases of personality and behavior (Vol. 2, pp. 43-58). New York: Hemisphere Publishing. Buss, A. H., \& Plomin, R. (1975). A temperament theory of personality development. New York: Wiley.

CoREN, S. (1988). Prediction of insomnia from arousability predisposition scores: Scale development and cross-validation. Behavior Research \& Therapy, 26, 415-420.

Coren, S., \& AKs, D. J. (1990). Prediction of task-related arousal under conditions of environmental distraction. Manuscript submitted for publication.

Danilova, N. N. (1985). Dynamics of AEP and heart rate as indicators of individual arousal level and learning. In J. Strelau, F. H. Farley, \& H. J. Eysenck (Eds.), The biological basis of personality: Vol. 1. Theories, measurement techniques and development (pp. 59-72). New York: Hemisphere Publishing.

EYSENCK, H. J. (1970). The structure of human personality. London: Methuen.

Kohn, P. M., Cowles, M. P., \& LAfreniere, K. (1987). Relationships between psychometric and experimental measures of arousability. Personality \& Individual Differences, 8, 225- 231.

Strelau, J. (1983). Temperament personality activity. London: Academic Press.

THAYER, R. E. (1967). Measurement of activation through self-report. Psychological Reports, 20, 663-678.

THAYER, R. E. (1970). Activation states as assessed by verbal reports and physiological variables. Psychophysiology, 7, 86-94.

UherIK, A. (1985). Personality and regulatory functions. In J. Strelau, F. H. Farley, \& A. Gale (Eds.), The biological bases of personality and behavior (Vol. 2, pp. 97-106). New York: Hemisphere Publishing.

(Manuscript received April 13, 1990.) 